Int. J. Speleol. 4 (1972), pp. 9-18.

\title{
Une nouvelle espèce africaine du genre Stenasel/us (Crustacea Isopoda Asellota) du bassin du Niger
}

\author{
J.A. BIRSTEIN *
}

La première espèce africaine du genre archaique Stenasellus (St. chappuisi Remy) a été décrite du puits du poste de Man (Côte-d'Ivoire) (Remy, 1938) et a été retrouvée dans un puits de Guinée portugaise (Braga, 1950). Plus tard le même puits de Côte-d'Ivoire a fourni la deuxième espèce, St. africanus Monod (Monod, 1945) et le puits de Guinée portugaise la troisième espèce, St. guineensis Braga; malheureusement le mâle de cette dernière espèce est encore inconnu (Braga, 1950). Plus tard, trois espèces (St. leleupi Chappuis, St. congolensis Chappuis et $S t$. dartevellei Chappuis) ont été découvertes dans le bassin du Congo (Chappuis, 1951; 1952) et enfin la dernière espèce (St. pardii Lanza) en Somalie (Lanza, 1966).

Ces faits manifestent la richesse de l'Afrique équatoriale en Stenasellus, mais toutes les espèces n'étaient connues jusqu'à maintenant qu'en Côte-d'Ivoire, dans le bassin du Congo et en Somalie.

Etudiant la faune des puits du bassin du Niger en février 1966 V.N. Andronov a récolté dans l'un d'eux situé dans la ville de Sicascau (Mali) 14 mâles et 10 femelles de Stenasellus, qu'il a bien voulu me confier pour étude, ce dont je le remercie vivement. Je décris ci-dessous une nouvelle espèce pour ces spécimens.

\section{Description de Stenasellus laticarpus n.sp.}

Type de l'espèce - $\delta^{\star}$ - longueur $10,1 \mathrm{~mm}$.

Corps allongé, à bords parallèles, environ $5 \frac{1}{2}$ fois plus long que large.

Tête trapézoidale, $1 \frac{1}{2}$ fois plus large que longue, plus longue que le péréionite $I$, bord frontal de celui-ci concave.

Péréionites I-IV de longueurs subégales, péréionite V plus court, péréionite VII plus long. Epines marginales peu nombreuses. Les angles postérieurs des péréionites I-IV avec une rangée de petites épines. Les coxas de péréionites I-VII sont visibles dorsalement.

Pléonites I-II bien développés, chacun un peu plus court que la moitié de la longueur de péréionite VII.

Pléotelson subrectangulaire, sa longueur représente $1 / 5$ de celle du corps entier et surpasse un peu sa largeur.

Antennules plus longues que la hampe de l'antenne. La longueur relative des

* La rédaction a l'immense regret de vous faire part du décès du Professeur Birstein, survenu à Moscou le 8 juillet 1970; elle espère pouvoir publier dans un très proche avenir, un article consacré à la mémoire de l'illustre biologiste russe, fondateur et promoteur de la série Biospeologica sovietica. 
articles de leurs hampes est respectivement 33:27:15. Le premier article est large et un peu courbe. Le fouet comporte 15 articles, dont les 10 distaux portent des lames olfactives, plus courtes que les articles respectifs.

Antennes dépassant le péréionite IV. La longueur relative des 5 articles distaux de leurs hampes est respectivement 7:43:22:44:70. Le fouet comporte 40 articles

Maxillules: le lobe interne porte 3 grandes tiges barbelées et 2 plus petites; la proportion de leurs longueurs analogue à celle de St. africanus Monod (Magniez, 1966).

Maxillipèdes dont le rétinacle comprend 4 crochets d'un còté et 3 crochets de l'autre.

Péréiopodes I: propodite environ $1 \frac{1 / 2}{2}$ fois plus long que large, à bord sternal légèrement concave avec 13 tiges semi-barbelées dont 2 phanères ensiformes. La rangée submarginale caudale comporte 10 soies lisses, dactylopodite avec rangée sternale de 13 épines et 4 soies, carpopodite avec 10 épines à la rangée sternale et 4 soies longues et grêles insérées en direction distale de cette rangée.

Péréiopodes II-IV de structure semblable. Leur carpopodite est très large, de forme ovoïde, seulement 2 fois plus long que large, avec le bord sternal convexe, garni d'une rangée partiellement double de fortes épines courbées. Le péréiopode II possède 23 , le péréiopode III 20 et le péréiopode IV 23 de ces épines. Le nombre des épines du bord sternal des propodites est respectivement 6 , 7 et 8 . Le dactylopodite de ces péréiopodes porte 2 épines sternales.

Péréiopodes V-VII avec carpopodites étroits de structure normale et 2 épines dactyliennes.

Pléopodes I: protopodite pentagonal, un peu plus large que long, à marge externe convexe formant un angle droit, marge interne rectiligne, sans rétinacle, et continuée en direction distale par une apophyse triangulaire. Exopodite subovalaire, environ $1 \frac{1}{2}$ fois plus long que large, pas plus étroit que le protopodite; ses marges interne, distale et latéro-externe sont garnies de 37 tiges lisses dont les tiges de la marge interne sont plus longues.

Pléopodes II: protopodite quadrangulaire, marges interne et externe subrectilignes, angles arrondis. Exopodite 2 fois plus court que le protopodite; article distal subtrapézoidal, plus large distalement, aussi long que large, portant une rangée de 6 soies courtes, lisses à l'angle distal externe et quelques petits crochets au bord distal interne. Endopodite presqu'aussi long que le protopodite; article proximal court, trapu, très large distalement; article distal environ 3 fois plus long que l'article proximal, isodiamétrique à extrémité obtuse avec un vaste orifice.

Pléopodes III: exopodite environ 2 fois plus long que large, article distal aussi long et large que l'article proximal à 9 tiges courtes sur sa marge distale; endopodite atteignant les $3 / 4$ de la longueur de l'exopodite mais 2 fois plus étroit.

Pléopodes IV: exopodite ovalaire, plus de 2 fois plus long que large, à deux sutures obliques, une proximale qui commence à la marge interne et une distale qui commence à la marge externe; tous les deux n'atteignent pas la marge opposée; les marges interne et distale portent 39 tiges ciliées, marge externe finement ciliée; endopodite 2 fois plus étroit et un peu plus court que l'exopodite.

Pléopodes V: exopodite également ovalaire, 2 fois plus long que large, subdivisé 


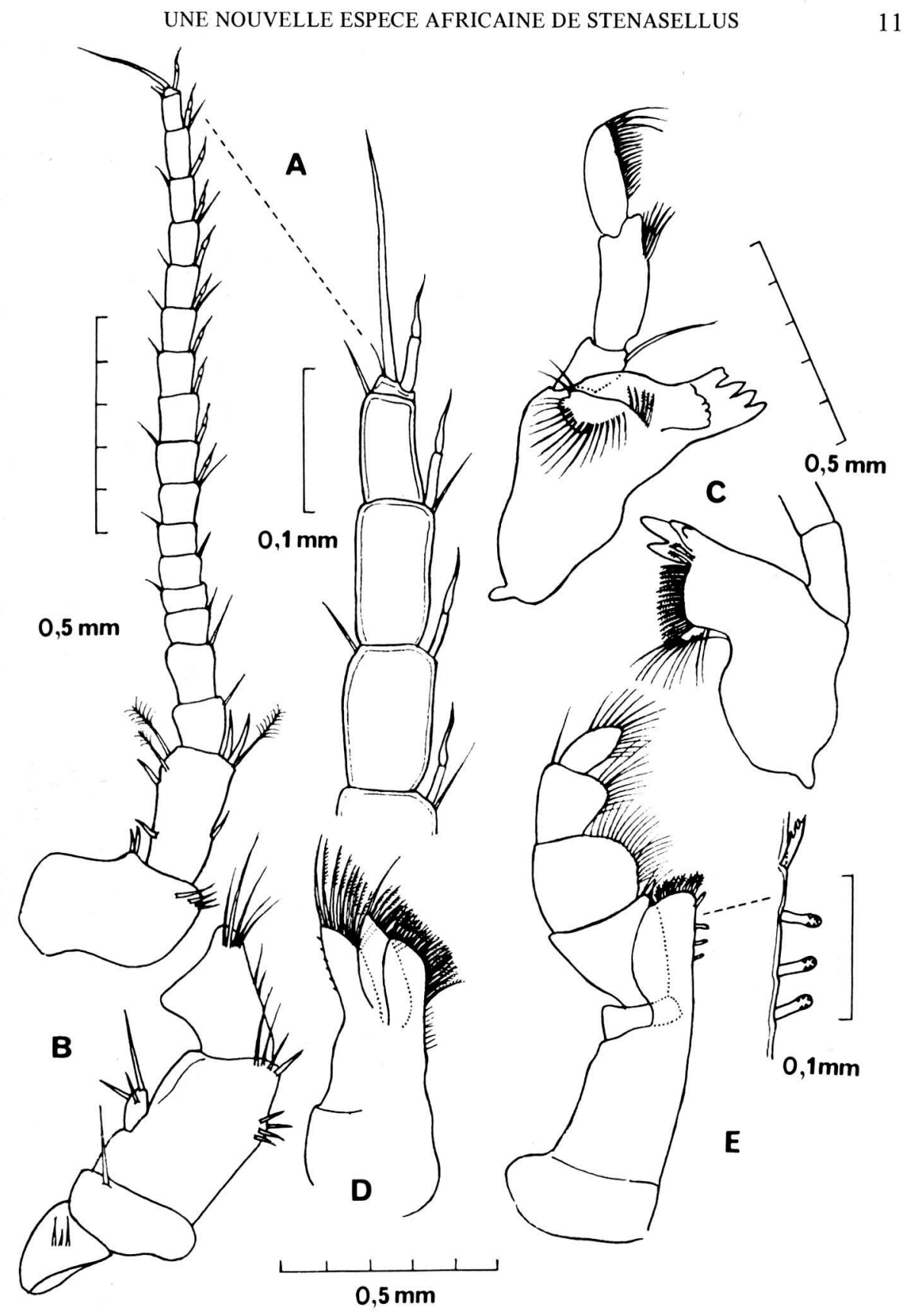

Planche 1: Fig. A: Antennule. Fig. B: Hampe de l'antenne. Fig. C: Mandibles. Fig. D: Maxillule. Fig. E: Maxillipède. 


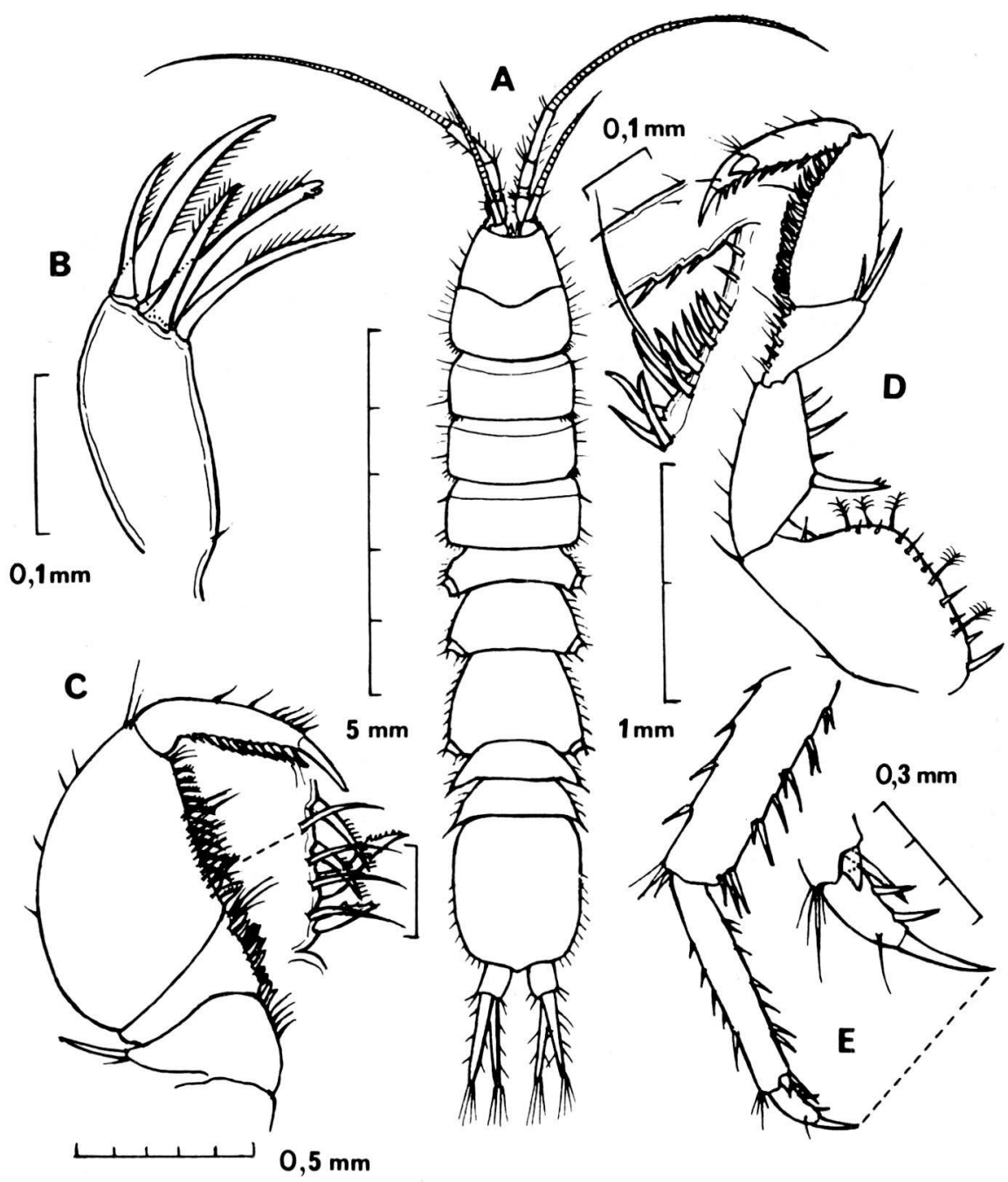

Planche 2: Fig. A: Mâle, face dorsale. Fig. B: Lame interne de la maxillule. Fig. C: Extrémité du péréiopode I d'un o.|Fig. D: Péréiopode II d'un ơ.|Fig. E: Extrémité du péréiopode VII d'un ơ. 
par une suture complète et oblique en deux articles dont l'article distal est environ 2 fois plus petit que le proximal; la marge interne garnie de rangée de fins denticules; endopodite $1 \frac{1}{2} 2$ fois plus étroit et un peu plus court que l'exopodite.

Uropodes un peu plus courts que le pléotelson, leurs exopodites aussi longs que les endopodites, 2 fois plus longs que les protopodites.

† - longueur $10,5 \mathrm{~mm}$.

Corps comme chez le mâle.

Antennules: fouet avec 14 articles à lames olfactives sur les 10 articles.

Antennes: fouet avec 39 articles.

Péréiopodes I et V à VII pratiquement identiques à ceux du mâle.

Péréiopodes II-IV: carpopodites plus étroits que ceux du mâle, $2 \frac{1 / 2}{2}$ fois plus longs que larges, à bord sternal moins convexe, garni d'épines peu nombreuses: péréiopode II portant 12 , péréiopode III et IV 15 épines chacun. Le bord sternal du propodite est muni de 5 épines.

Pléopodes II libres, subtriangulaires, un peu plus longs que large, leur marge interne est rectiligne et glabre. Ils portent 3 soies lisses sur l'angle distal et 1 soie au milieu de la marge externe. L'angle proximal externe est arrondi et garni de petits denticules.

\section{REMARQUES SYSTÉMATIQUES}

St. laticarpus n. sp. se distingue de toutes les 25 espèces connues du genre Stenasellus (s.1.) par la structure originale des carpopodites des péréiopodes II à IV. Ceux-ci sont élargis et munis d'épines courbes multiples à leur bord sternal. L'intérêt particulier de ce caractère résulte du fait qu'il montre un notable dimorphisme sexuel: le mâle possède des carpopodites plus larges avec des épines plus nombreuses en comparaison de ceux de la femelle. Dans toutes les diagnoses du genre Stenasellus (Racovitza, 1924, 1950; Birstein, 1951; Magniez, 1966) on souligne l'absence de dimorphisme sexuel dans la structure de péréiopodes chez les représentants de ce genre. Sous ce rapport St. laticarpus n. sp. est la seule exception.

Les autres caractères distinctifs observés chez St. laticarpus montrent que cette espèce doit être rangée parmi les espèces groupées autour de St. gjorgjevici Racovitza, caractérisées principalement par l'extrémité obtuse de l'endopodite du pléopode II du mâle. Ce groupe renferme St. gjorgjevici Racovitza, St. hungaricus (Méhely), St. bureschi Racovitza, St. lakatnicensis Buresch et Gueorguiev St. nuragicus Argano et St. assorgiai Argano. La nouvelle espèce se différencie de toutes les espèces citées par son exopodite du pléopode I du mâle assez large.

Tout porte à croire que nous devrions aussi inclure dans ce groupe St. africanus Monod, pour lequel Lanza (1966) a créé un genre indépendant Magniezia en se basant essentiellement sur la structure de l'endopodite bilobé du pléopode II du mâle. Mais les études détaillées de Cvetkov (1967) ont montré la ressemblance 


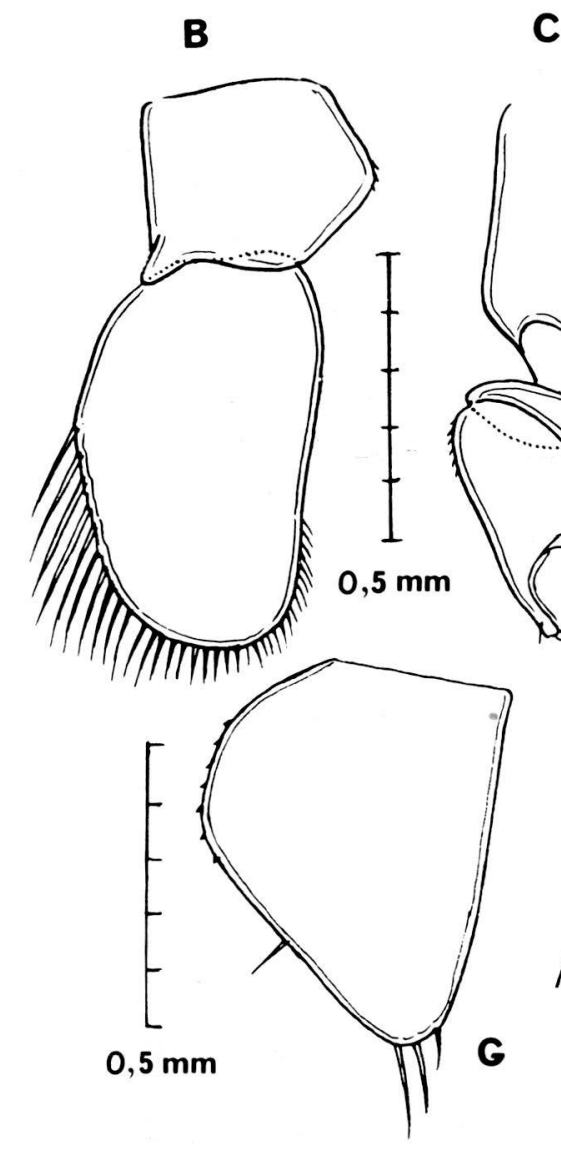

C
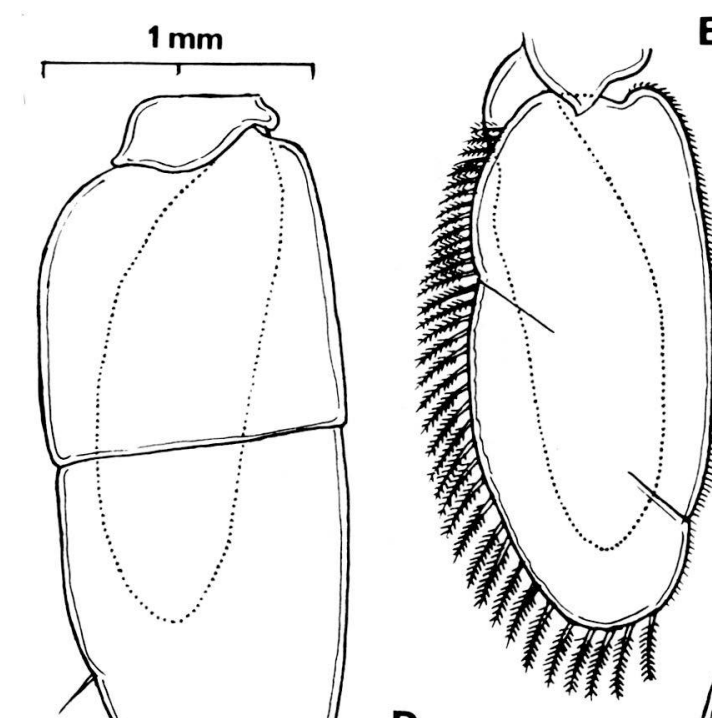

$\mathbf{E}$

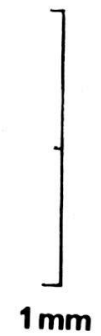

$1 \mathrm{~mm}$

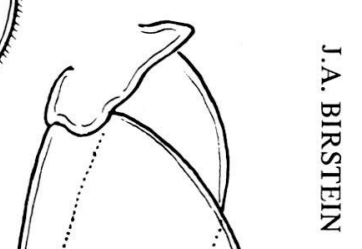

Planche 3: Fig. A: Péréiopode II d’une \%. Fig. B: Pléopode I d’un ơ. Fig. C: Pléopode II d’un ơ. Fig. D-E: Pléopodes III-V d’un ơ. Fig. G: Pléopode II d’une 9. 
principale sous ce rapport entre St. africanus Monod et St. bureschi Racovitza (et les autres espèces de ce groupe). Selon Cvetkov "l'organe copulateur de St. bureschi présente une feuille bilobée, enroulée mais aux bords non coalescents, de l'article II de l'endopodite. Traitée à l'hydrate potassique, elle peut se déployer" (p. 163). Les autres caractères du genre Magniezia mentionnés dans sa diagnose n'ont pas non plus de valeur générique. Comme St. africanus Monod St. laticarpus possède 5 tiges fortes subégales au lobe maxillulaire interne et 4 crochets au rétinacle des maxillipèdes.

St. laticarpus est plus éloigné des autres espèces africaines que de St. africanus Monod, d'autant que St. laticarpus Monod sont géographiquement voisines. Trois espèces congolaises (St. leleupi Chappuis, St. congolensis Chappuis et St. dartevellei Chappuis) sont très proches l'une de l'autre et indiscutablement apparentées (Chappuis, 1950, 1951; Magniez, 1966). Pour ces espèces Magniez (1966) a créé le genre Metastenasellus qui peut se distinguer des autres Stenasellinae par l'endopodite du pléopode II du mâle uniarticulé, constitué selon un processus d'enroulement en hélice, et aussi par l'absence du rétinacle au protopodite du pléopode I du mâle et par les pléopodes II de la femelle soudés sur leur partie proximale. Selon Magniez "toutes ces formes africaines, sauf africanus, présentent des variations structurales très importantes par rapport au type, sans aucune mesure avec celles qui affectent des espèces, européennes entre elles” (p. 175). D’après Magniez, St. skopjensis Karaman et les formes voisines appartiennent à un groupe qui a "article proximal de l'endopodite relativement court et trapu" (p. 178). En effet, chez St. skopljensis "der Innenast der II Pleopoden nicht gelenkig, wie bei Stenasellus sonst, sondern beide Glieder miteinander festverwachsen, wie bei Asellus s. str." (Karaman, 1948, p. 9) et chez St. skopljensis croaticus Karaman" das Gelenk im Innenaste ist aber trotzdem hier wie auch beim typ. skopljensis und $f$. meridionalis festverwachsen, nicht gelenkig wie z.B. beim St. gjorgjevici" (Karaman, 1954, p. 10).

A mon avis, la structure des organes copulateurs des espèces congolaises rappelle celle qui a été observée chez les deux espèces européennes, St. skopljensis Karaman [la forme typique et les deux formes meridionalis Karaman et croaticus Karaman (Karaman, 1948, 1954)] et St. rumelicus Cvetkov (Cvetkov, 1967) qui a lui aussi l'endopodite de pléopode II du mâle uniarticulé à "l'apex ... enroulé en colimaçon" (Cvetkov, loc. cit., p. 164). Sous ce rapport on ne peut constater aucune différence principale entre les espèces congolaises et européennes citées mais nous ne savons pas encore s'il faut considérer cette ressemblance comme une convergence morphologique ou comme l'indice de la parenté directe.

St. skopljensis Karaman et St. rumelicus Cvetkov se distinguent d'autre part des espèces congolaises ayant un rétinacle sur le protopodite du pléopode I du mâle, les pléopodes II de la femelle indépendants et les endopodites des pléopodes III, IV et $\mathrm{V}$ bilobés comme chez les deux autres espèces africaines, St. chappuisi Remy et St. pardii Lanza.

L'absence de rétinacle au protopodite du pléopode I du mâle n'a pas de valeur générique. Le bord interne de cet article est privé d'épines chez plusieurs espèces de Stenasellus par exemple chez St. africanus Monod, St. laticarpus, St. breuili Racovitza, St. asiaticus Birstein et Starostin et St. lakatnicensis Buresch et 
Gueorguiev. Ainsi la seule particularité de trois espèces congolaises sont les pléopodes II de la femelle soudés entre eux sur leur partie proximale, mais ce caractère et probablement insuffisant pour la définition d'un genre indépendant (Metastenasellus).

Outre Metastenasellus Magniez a créé un autre genre africain Parastenasellus pour St. chappuisi Remy. Cette espèce présente en effet des caractères très particuliers, notamment la présence de l'épipodite au maxillipèce du mâle, les courts pléonites et quelques particularités des deux premières pléopodes du mâle, mais des endopodites bilobés existent aussi aux pléopodes III, IV et V chez St. skopljensis Karaman, St. rumelicus Cvetkov et St. pardii Lanza; la constitution des exopodites de ces appendices de $S t$. chappuisi Remy évoque celle de $S t$. laticarpus. D'autre part $S t$. chappuisi Remy montre quelques traits de ressemblance avec une autre espèce africaine St. pardii Lanza, en particulier en ce qui concerne l'aspect de l'endopodite du pléopode II du mâle et des pléopodes II à V. Cette dernière espèce ne présente elle aussi qu'une seule épine sur le dactyle des péréiopodes II à VII. D'après la structure et l'aspect de l'endopodite du pléopode II du mâle St. pardii Lanza doit être rangé entre St. chappuisi Remy et des représentants du groupe des espèces à terminaison aiguë.

On voit donc qu'aucun des genres endémiques africains des Stenasellinae fondés par Magniez et Lanza ne peut être déterminé par l'ensemble de caractères propres à chacun d'eux. Dans ces conditions il semble donc préférable de conserver pour le moment l'unité du genre Stenasellus sensu lato. Pourtant il est possible d'y distinguer plusieurs groupes cohérents d'espèces (chacun défini par la constitution de l'endopodite du pléopode II du mâle) dont certains ont été déjà établis par Magniez (1966) et Cvetkov (1967).

1. Le groupe de St. gjorgjevici Racovitza. Article proximal environ ou plus de 2 fois plus court que l'article distal. Article distal isodiamétrique ou un peu plus large distalement à terminaison (avec bilobation plus ou moins visible), sans crochets. Ce groupe renferme St. africanus Monod, 1945, St. assorgiai Argano, 1968, St. bureschi Racovitza, 1950, St. gjorgjevici Racovitza, 1924 avec sa sous-espèce robustus (Méhely, 1927)*, St. hungaricus (Méhely) avec sa sousespèce thermalis Mestrov, 1960, St. lakatnicensis Buresch et Gueorguiev, 1962, St. laticarpus n. sp. et St. nuragicus Argano, 1968.

2. Le groupe de St. breuili Racovitza. Articles proximal et distal subégaux. Article distal isodiamétrique, à terminaison obtuse, muni de crochets à la marge externe. St. breuili Racovitza, 1924, St. galhanoae Braga, 1962.

3. Le groupe de St. virei Dollfus. Article proximal un peu plus long que la moitié de l'article distal. Les marges interne et externe de l'article distal convexes, la terminaison aiguë. St. asiaticus Birstein et Starostin, 1949, St. buili Remy, St. nobrei Braga, 1942, St. racovitzai Razzauti, 1925, St. virei Dollfus, 1897 avec ses 5

* Selon Méhely (1927, p. 19) "diese neue Unterart ähnelt betreffs des I. und II. Pleopodits des Männchens viel mehr dem von Racovitza beschriebenen ... serbischen Stenasellus Gjorgjevici, als der Abaligeter Protelsonia hungarica". Pr. hungarica robusta Méhely est insuffisamment décrite mais peut-être probablement interprétée comme une sous-espèce de St. gjorgjevici Rac. (Birstein, 1951). 
sous-espèces virei Dollfus, 1897, hussoni Magniez, 1968, buchneri Stammer, 1936, angelieri Magniez, 1968 et boui Magniez, 1968.

4. Le groupe de St. chappuisi Remy. Article proximal plus long que la moitié de l'article distal. La marge externe de l'article distal presque rectiligne, la marge interne convexe, sa moitié distale se rétrécit progressivement vers la terminaison. St. chappuisi Remy, 1938, St. pardii Lanza, 1966.

5. Le groupe de St. skopljensis Karaman. Endopodite unisegmenté, à terminaison enroulée en colimaçon. Ce groupe comprend St. congolensis Chappuis, 1951, St. dartevellei Chappuis, 1952, St. leleupi Chappuis 1951, St. rumelicus Cvetkov, 1967, St. skopljensis Karaman, 1936, avec ses sous-espèces croaticus Karaman, 1954 et meridionalis Karaman, 1954.

A l'heure actuelle il est préférable de ne pas attribuer à ces groupes la valeur générique ou sous-générique car ils sont basés seulement sur un caractère unique. Mais un autre point reste à signaler: la répartition géographique vaste et discontinue des groupes de St. gjorgjevici Racovitza et de St. skopljensis Karaman, l'un le moins et l'autre le plus spécialisé, qui occupent le Sud de l'Europe et l'Afrique équatoriale; par contre les groupes de St. breuili Racovitza et de St. virei Dollfus habitent seulement le Sud de 1'Aurope ou l'Eurasie et le groupe de St. Chappuisi Remy est localisé en Afrique équatoriale. Ainsi les premiers groupes ont conservé leur unité sur de grands espaces et probablement durant une longue période géologique.

\section{RÉ S U M É}

En Afrique les représentants du genre Stenasellus s.l. étaient jusqu'à présent connus en Côte-d'Ivoire, dans le bassin du Congo et en Somalie. La nouvelle espèce de ce genre, St. laticarpus, a été découverte dans le bassin du Niger dans un puits de la ville de Sicascau (Mali). Cette espèce se distingue de toutes les autres par les carpopodites des péréiopodes II à IV élargis et munis de multiples épines courbes à leur bord sternal. Ce caractère montre un dimorphisme sexuel notable. D'après d'autres caractères elle peut être rangée parmi les espèces groupées autour de $S t$. gjorgjevici Racovitza et présente quelques affinités avec $S t$. africanus Monod.

Il semble préférable de conserver en attendant 1'unité du genre Stenasellus. La validité des genres endémiques africains Metastenasellus Magniez, Parastenasellus Magniez et Magniezia Lanza demande à être confirmée.

\section{S U M M A R Y}

The representatives of the genus Stenasellus (sensu lato) in Africa have been previously known from Côte d'lvoire, bassin of the Congo and Somali. The new species of this genus was collected from the well in the town Sicascau (Mali). This species differs from all others in the structure of the carpopodites of pereopods II-IV which are enlarged and armed with multiple curved spines. This character shows a distinct sexual dimorphism. In all other features $S t$. laticarpus sp.n. belongs 
to the group of St. gjorgjevici Rac. and shows the closest resemblance to St. africanus Monod.

It is now preferable to conserve the unity of the genus Stenasellus (sensu lato). The validity of the African endemic genera Metastenasellus Magniez, Parastenasellus Magniez and Magniezia Lanza needs to be confirmed.

\section{B I B L I O G R A P H I E}

ARGANO, R. 1968. Due nuovi Stenasellus di Sardegna (Crustacea Isopoda Asellota). Fragm. entomol., 6: 1-22.

BIRSTEIN, J.A. 1951. Asellota. Faune de l'URSS, VII/5, Moscou : 1-142.

BRAGA, J.M. 1942. Un Isopode nouveau du Portugal: Stenasellus Nobrei n.sp. Mem. Mus. Zool. Univ. Coîmbra, 132: 1-2.

BRAGA, J.M. 1950. Sur deux Stenasellus (Crust. Isopoda) de la Guinée Portugaise. An. Fac. Cien. Porto, 35: 50-56.

BURESCH, I. et GUEORGUIEV, V. 1962. Contribution à la connaissance des espèces du genre Stenasellus (Crustacea, Asellota) en Bulgarie avec description d'une nouvelle espèce. Bull. Inst. Zool. et Musée, II: 189-206.

CHAPPUIS, P.A. (1951). Isopodes et Copépodes cavernicoles. Rev. Zool. Bot. Afr., 44: 342-359.

CHAPPUIS, P.A. (1952). Un nouveau Stenasellus du Congo belge. Rev. Zool. Bot. Afr., 45: 353-357.

CVETKOV, L. 1967. Au sujet de la faune des Stenasellus de la Péninsule Balkanique et sur l'origine des Stenasellus. Bull. Inst. Zool. et Musée Acad. Bulg. Sci., 23: 139-165.

KARAMAN, St. 1936. Ein Stenasellus aus dem Grundwasser von Skoplje, St. gjorgjevici skopljensis n. subsp. Glasnik (Bull.) Soc. Scient. Skoplje, 17: 137-141.

KARAMAN, St. (1984). Über das Männchen von Stenasellus skopljensis Karaman. Prirodosl. istraz. Jugosl. Akad. Znatn., 24: 5-10.

KARAMAN St. (1954). Über die jugoslavischen Stenasellus Arten. Fram. Balc. Mus. Maced Sci. Nat., 1: 7-20.

LANZA, B. (1966). Stenasellus pardii sp. n. della Somalia e note sistematiche su gli Stenasellinae (crustacea, Isopoda). Mon. Zool. Ital., 74: 221-256.

MAGNIEZ, G. 1966. Contribution à la systématique des Stenasellinae d'Afrique (Crustacés, Asellotes). Int. J. Speleol., 2: 173-190.

MAGNIEZ, G. 1968. L'espèce polytypique Stenasellus virei Dollfus, 1897 (Crustacé Isopode hypogé). Ann. spéléol., 23: 363-407.

MEHely, L. 1924. Protelsonia hungarica nov. gen., n. sp., ein blinder Isopode aus Ungarn. Zool. Anz., 58: 353-357.

MEHELY, L. 1927. Neue Würmer und Krebse aus Ungarn. Budapest: 1-19.

MESTROV, M. 1960. Stenasellus hungaricus thermalis sp. n. (Crustacea Isopoda). Fund einer vorglazialen Art in warmen Quellen bei Zagreb. Biol. Glasn., 13: 345-350.

MONOD, Th. 1945. Un nouveau Stenasellus ouest-africain. Bull. Inst. Franc. Afr. Noire, 7: $101-114$

RACOVITZA, E.G. 1924. Diagnoses des genres Asellus et Stenasellus et description de deux Stenasellus nouveaux (Note préliminaire). Bull. Soc. Stiin. Cluj, 2: 81-92.

RACOVITZA, E.G. 1950. Asellides. Première série: Stenasellus. Arch. Zool. exp. gén., 87: $1-94$.

RAZZAUTI, A. 1925. Sopra un nuovo crostaceo troglobio italiano (Stenasellus racovitzai). Comunicazione preliminare. Atti Mem. R. Ac. Petrarca Sc. Lett. Arti. (n.ser.), 5-28.

REMY, P. 1938. Un Stenasellus en Afrique occidentale française: Stenasellus chappuisi n. sp. de la Côte-d'Ivoire. Arch. Zool. exp. gén., 79: 69-74.

REMY, P. 1949. Stenasellus buili n.sp. de la grotte de la Giraudasso à Soulatgé, Aude. Bull. Soc. Linn., Lyon, 18: 153-157. 